\title{
MANOBRA DA COMPRESSÃO DO SULCO CERVICAL
}

\author{
Toribia Mottos* \\ Wanda Escobar da Silva Freddi** \\ Nelson Rodrigues Júnior ***
}

MOTTOS, T.; FREDDI, W. E. da S.; RODRIGUES JUNIOR, N. Manobra da compres-

são do sulco cervical. Rev. Esc. Enf. USP, São Paulo, 15(2):183-192, 1981.

Estudo sobre a utilização da manobra de compressão do sulco cervical fetal, em parturientes, como meio para o diagnóstico de circular cervical do cordão umbilical.

$A$ população deste estudo foi constituída por 132 parturientes (58 primíparas e 74 multiparas), com feto único em apresentação cefálica, no período de dilatação, com membranas amnióticas integras e sem sinais clinicos de sofrimento fetal.

Os dados obtidos demonstram a eficácia da manobra para a deteç̧ão da presença de circular cervical do cordão umbilical.

\section{INTRODUÇÃO}

0 presente trabalho pretende aquilatar a validade da manobra da compressão do sulco cervical, para estabelecer o diagnóstico de circular cervical do cordão umbilical.

Esse diagnóstico é deveras difícil de ser feito durante o trabalho de parto. $\mathrm{Na}$ maioria das vezes, só pode existir suposição da sua presença.

Do ponto de vista prático, na assistência ao parto, é importante o diagnóstico de circular pelas repercussões que essa patologia do funículo pode acarretar, como o sofrimento fetal e, conseqüente, aumento da morbi-mortalidade perinatais.

Com a possibilidade da circular cervical ser diagnosticada durante o trabalho de parto, o profissional que assiste à parturiente pode prevenir a anoxia fetal grave.

Não encontramos, na literatura médica, trabalho científico que possa valorizar meios propedêuticos que denunciem a circular cervical do ordão umbilical.

DE LEE \& GREENHILL ${ }^{4}$ afirmam que as circulares do cordão umbilical, em torno do feto, têm repercussões somente quando existe comprimento anormalmente pequeno do cordão (brevidade do cordão umbilical), ou quando as circulares são apertadas, vindo comprometer a circulação fetal. Segundo os autores, as circulares cervicais do cordão estão presentes em um quinto dos fetos, podendo intervir no mecanismo do parto e causar apresentações defletidas, retardar a rotação interna da cabeça fetal ou das espáduas e causar situação transversa. Ressaltam ainda que a compressão prolongada do cordão umbilical entre o pescoço do

* Professor Assistente da disciplina Enfermagem Obstétrica e Neonatal da EEUSP.

* * Professor Assistente Doutor da disciplina Enfermagem Obstétrica e Neonatal da EEUSP.

* * Estudante do 6.0 ano do Curso de Graduação em Medicina da Escola Paulista de Medicina. 
feto e o pube da mãe, pode causar a anoxia fetal, o que se verifica especialmente em primíparas. Para eles, algumas vezes durante o parto, pode-se ouvir nitidamente um sopro funicular na região cervical e um batimento fetal lento, revelado pela pressão digital.

Segundo PEREZ ${ }^{9}$ o comprimento do cordão umbilical varia entre 50 e $55 \mathrm{~cm}$; quando não atinge $20 \mathrm{~cm}$ existe brevidade real do cordão umbilical. Esta brevidade pode ser aparente, acidental ou relativa nos casos das circulares do cordão. A etiologia das circulares é muito discutida; segundo este autor, as circulares ocorrem nos primeiros meses de gestação, devido aos movimentos fetais, sendo sua freqüência de 20 a $25 \%$. Cita ainda o mesmo que, as circulares do cordão umbilical podem ocasionar, durante o trabalho de parto, os seguintes sintomas: contrações irregulares, periodo expulsivo prolongado e dor mais intensa em um ponto fixo do útero. A brevidade do cordão umbilical mais acentuada, além dos sintomas já descritos, pode ocasionar ainda: contrações uterinas espasmódicas, dilatação cervical insidiosa, falta de insinuação ou progressão fetal e, por último sofrimento fetal sem motivo claro que o explique; afirma, também, que o diagnóstico de circular cervical do cordão umbilical, durante o trabalho de parto, é impossível, podendo ser suspeitada através da progressão difícil ou detida do feto e de sinais precoces de sofrimento fetal. $O$ prognóstico fetal é grave pelo perigo de anoxia por interrupção circulatória feto-placentária.

GRELLE ${ }^{5}$ menciona que a porcentagem de circular do cordão umbilical é de 20 a $25 \%$, sendo que em cerca de $95 \%$ localiza-se em torno do pescoço do feto e, mais raramente, ao redor do tronco e dos membros. Como fator etiológico considera, em primeiro plano, o comprimento exagerado do cordão e, em segundo, o excesso de mobilidade fetal (feto pequeno e líquido amniótico abundante). Nas circulares cervicais do cordão umbilical, durante o trabalho de parto, podem ocorrer as seguintes complicações: deflexão cefálica (apresentação de face ou de fronte), rotura do cordão umbilical, descolamento prematuro da placenta e anoxia fetal decorrente da compressão do cordão umbilical, à medida que a flexão cefálica se acentua. As circulares apertadas sob ação das contrações podem causar morte fetal durante o trabalho de parto.

$\mathrm{HON}^{8}$ comenta que é possível provocar desaceleração variável dos batimentos cardíacos fetais se for aplicada pressão sobre o feto, através da parede abdominal materna, com a seguinte técnica: os polos superior e inferior do feto são comprimidos com ambas as mãos através da palpação abdominal; a pressão é exercida em várias direções, para verificar se o cordão umbilical está comprimido entre o feto e as estruturas maternas; quando isto ocorre há uma mudança na freqüência cardíaca fetal.

GREENHILL ${ }^{6}$ refere cinco tipos de patologia do cordão umbilical, dentre as quais a ocorrência de circulares numa porcentagem de 12,6 a $32,6 \%$ de todos os nascimentos. Cita o mesmo que "infelizmente não é possivel detectar clinicamente os sinais precoces de circulares do cordão umbilical".

Segundo GULIN 7 "a compressão da cabeça e fontanela fetais provoca quedas da freqüência cardíaca fetal de caracteres similares aos dos dips tipo $I$, isto é: 
a) a curta latência entre a compressão e a queda da freqüência cardíaca fetal;

b) rapidez da quoda da freqüência cardiaca-fetal;

c) rápida recuperação, embora a compressão continue, o que configura o fenômeno do "escape" caracteristico dos efeitos vagais do feto.

A compressão do cordão umbilical provoca quedas da freqüência cardíaca fetal, similares aos dips tipo I;

Os dips tipo I aparecem habitualmente na segunda metade do parto, quando a cabeça fetal está sujeit a a maior compressão pelas contrações uterinas".

Nesta pesquisa, a compressão do cordão umbilical foi obtida exercendo-sc pressão com ambas as mãos entre a cabeça e os ombros do feto, em parturientes em início de trabalho de parto com membranas amnióticas íntegras.

ARAMBURÚ et alii ${ }^{2}$ ressaltam que os dips tipo I são mais freqüentes nos fetos de parturientes em trabalho de parto avançado e com membranas amnióticas rotas.

BARCELLOS \& NAHOUM ${ }^{3}$ citam que as circulares de cordão umbilical são comuns em torno de $20 \%$ dos partos e quase sempre cervicais; outras vezes estão ao redor do tronco ou dos membros. Na maioria dos casos são únicas; ocasionalmente duplas; raramente triplas e excepcionalmente quádruplas. Os cordões longos (quando ultrapassam uma vez e meia o comprimento do feto) e as condições que exageram a mobilidade do concepto (feto de dimensões reduzidas e aumento do volume o liquido amniótico) propiciam circulares. Dizem os autores que geralmente não há repercussões patológicas, mas podem condicionar brevidade relativa, apresentações distócicas (de face e de fronte), anoxia e óbito fetal, principalmente durante o periodo expulsivo.

PSCHYREMBEL ${ }^{10}$ informa que em média o comprimento do cordão umbilical é de $50 \mathrm{~cm}$, podendo oscilar entre 20 a $150 \mathrm{~cm}$. $O$ autor refere que o cordão umbilical curto ou muito longo pode ocasionar complicações tocológicas, como por exemplo, a asfixia do recém-nascido que é citada como uma das complicações provenientes da circular cervical do cordão umbilical, ocasionando a mesma transtornos da circulação feto-placentária. Quando à hipoxia intrauterina e a acidose, refere o autor que a forma clássica de transtorno intrauterino fetal é originada por qualquer motivo que produza, no sangue ou tecido, falta de oxigênio com sobrecarga de gás carbônico. Se este transtorno é pronunciado, ocasionará asfixia fetal. $O$ autor comenta que são muitas as causas deste transtorno e, dentre elas, como fetais, a circular cervical do cordão umbilical.

SHAFFER \& AVERY " salientam que "o cordão umbilical é uma estrutura de máxima importância para o feto, servindo como via única que fornece oxigênio c nutrição, e descarrega dióxido de carbono e produtos de queima. Qualquer acidente que diminua o fluxo sanguíneo através deste canal constitue um grave perigo para a vida ou para a saúde do feto ou do recém-nascido".

Referem os autores, que entre estes acidentes, destaca-se a compressão do cordão umbilical, ocasionado, por circulares em torno do corpo, em uma das extremi- 
dades ou no pescoço do feto. Assinalam, ainda, que a circular do cordão umbilical, em alguma parte do feto, não indica necessariamente que o fluxo sanguíneo esteja impedido. Em casos de circulares apertadas do cordão umbilical pode resultar uma verdadeira compressão, impedindo o fluxo sanguíneo materno-fetal. Acrescentam que uma circular cervical apertada prejudica tanto o feto como uma circular em torno do punho, quando o fluxo sanguíneo fica completamente obstruído. Em casos de circular cervical menos apertada é concebivel que possa haver impedimento do retorno venoso, sem diminuição do fluxo arterial. Como conseqüência, poderão ocorrer hemorragias petequiais e edema cerebral, que terão significação maior do que as alterações congestivas semelhantes em uma das extremidades.

\section{OBJETIVOS}

- Verificar se a compressão do sulco servical pode ser uttlizada para o diagnóstico da circular cervical do cordão umbilical.

- Verificar se a paridade influi na resposta à manobra da compressão do sulco cervical.

- Verificar se existe correlação entre a resposta à manobra e a vitalidade do recém-nascido.

- Verificar se o comprimento do cordão umbilical pode influir na resposta à manobra da compressão do sulco cervical.

- Verificar se a tensão da circular cervical do cordão umbilical pode influir na resposta à manobra da compressão do sulco cervical.

\section{DEFINIÇÃO DE TERMOS}

Bradicardia fetal - freqüência cardíaca fetal inferior a 120 batimentos por minuto. a $20 \mathrm{~cm}$.

Brevidade do cordão umbilical - comprimento do cordão umbilical inferior

Dips $I$ - quedas transitórias da freqüência cardiaca fetal, provocadas por contrações uterinas, de decalage curta e de valor médio 3 segundos.

Manobra de Leopold - procura-se prender a cabeça fetal entre o polegar e o dedo médio, imprimindo-se movimentos de lateralidade que indicam o grau de penetração cefálica na bacia.

Resposta positiva à manobra - diminuição da freqüência dos batimentos cardíacos do feto (abaixo de 120).

Resposta negativa à manobra - batimentos cardíacos do feto inalterado.

Sulco cervical ou cefálico - sulco, resultante da inclinação da cabeça fetal sobre a espádua anterior.

Circular do cordão umbilical - Volta do cordão umbilical ao redor de uma parte do corpo do feto. 


\section{METODOLOGIA}

\section{População}

A população deste estudo foi constituida por 132 parturientes ( 58 primíparas e 74 multíparas) com feto único em apresentação cefálica, no periodo de dilatação, com membranas amnióticas íntegras e sem sinais clínicos de sofrimento fetal.

Acompanhamos essas parturientes até o $4 .^{c}$ periodo do parto, em maternidades que servem de campo de estágio de Enfermagem Obstétrica para os alunos da Escola de Enfermagem da USP.

\section{Instrumento de coleta de dados}

Utilizamos um formulário (anexo 1) com os seguintes itens: identificação da parturiente (idade, cor, estado civil), paridade, tipos de partos anteriores, controle dos batimentos cardíacos fetais (antes, durante e após a manobra), aspecto do líquido amniótico, tipo de parto atual, horas de trabalho de parto, condições do recém-nascido (avaliação pelo Método de Apgar, no $1 .^{\circ}$ e $5 .^{\circ}$ minutos de vida) e ocorrências relacionadas com o cordão umbilical. Os dados obtidos quanto aos batimentos cardíaco-fetais e a avaliação da vitalidade do recém-nascido foram anotados em dois itens especiais constantes no formulário.

\section{Método}

A manobra da compressão do sulco cervical foi realizada em parturientes com a bexiga vazia, no intervalo das contraçóes uterinas; as pacientes foram mantidas, durante a manobra, em decúbito dorsal, com o tronco ligeiramente erguido, pois o decúbito dorsal horizontal pode provocar hipotensão arterial e, conseqüentemente, anoxia fetal.

Localizamos o foco fetal com o estetoscópio de Pinard. Em seguida, com uma das mãos, fizemos a compressão do sulco cervical de tal maneira que o polegar ficasse em oposição aos outros quatro dedos, tal qual se faz na manobra de Leopold *, para verificação da mobilidade do polo cefálico. Evitamos, tanto quanto possível, a compressão da cabeça do feto, porque, segundo GULIN $^{7}$, esta pode determinar uma bradicardia fetal transitória.

Auscultamos os batimentos cardíacos do feto, durante e após a execução da manobra, para verificação da presença ou não de bradicardia fetal. Os resultados foram anotados no Formulário. A fim de evitar outras causas de erro, a pesquisa foi feita com parturientes durante o período de dilatação, com bolsa amnióica integra, para evitar os dips tipo I, que poderiam estar presentes. Auscultamos os batimentos cardíacos fetais cada 15 minutos, durante uma hora, antes e após realização da manobra.

Verificamos a presença ou a ausência de circular cervical do cordão umbilical, o número de voltas em torno do pescoço e o grau de tensão (apertada ou frouxa) durante o nascimento do feto.

\footnotetext{
* BRIQUET, 285.
} 
A avaliação da vitalidade do recém-nascido foi feita pelo método de Apgar ${ }^{1}$ no $10^{\circ}$ e no $5 .^{\circ}$ minuto de vida.

\section{RESULTADOS E COMENTÁRIOS}

\section{Características da população}

Idade: Das 132 parturientes que constituíram a população deste estudo, a maioria $(81,08 \%)$ encontra-se na faixa etária de 17 a 30 anos.

TABELA 1

Distribuição etária das 132 parturientes.

\begin{tabular}{l|rr}
\hline Idades & N.o de casos & \\
(em anos) & 9 & 6,81 \\
\hline até 16 & 107 & 81,08 \\
$17-30$ & 15 & 11.36 \\
$31-40$ & 1 & 0,75 \\
41 ou mais & 132 & 100,00 \\
\hline Total & 130 \\
\hline
\end{tabular}

Cor: A predominância foi de brancas $(50,00 \%)$ e a seguir pardas $(39,40 \%)$ e pretas $(10,60 \%)$.

TABELA 2

Distribuição das 132 parturientes segundo a cor.

\begin{tabular}{l|cr}
\hline \multirow{2}{*}{ Cor } & \multicolumn{2}{|c}{ Parturiente } \\
\cline { 2 - 3 } & N. & 50,09 \\
\hline Branca & 66 & 39,40 \\
Parda & 52 & 10,60 \\
Preta & 14 & - \\
Amarelas & - & 100,00 \\
\hline Total & 132 & 0 \\
\hline
\end{tabular}

Estado civil: Como podemos notar na tabela 3 , houve maior número de casadas $(58.33 \%)$.

TABELA 3

Estado civil das parturientes.

\begin{tabular}{l|cr}
\hline \multirow{2}{*}{ Estado Civil } & \multicolumn{2}{|c}{ Parturiente } \\
\cline { 2 - 3 } & N.0 & $\%$ \\
\hline Solteira & 55 & 41,67 \\
Casada & 77 & 58,33 \\
Viúva & - & - \\
Desquitada & - & - \\
\hline \multicolumn{1}{c|}{ Total } & 132 & 100,00 \\
\hline
\end{tabular}

Resposta à manobra.

A análise estatística dos dados apresentados na tabela 4 revela relação altamente significante entre a resposta positiva à manobra e a presença de circular cervical do cordão umbilical. Notamos que quando existe circular em torno do 
pescoço do feto a porcentagem de positividade da manobra foi de $79.60 \%$ e, quando há ausência de circular, a resposta negativa à manobra foi de $96,39 \%$.

Por outro lado, houve $10(20,40 \%)$ resultados falso positivos, isto é, resposta positiva à manobra com ausência de circular cervical do cordão umbilical, e 3 $(3,61 \%)$ resultados falso negativos, ou seja, resposta negativa à manobra com presença de circular cervical do cordão umbilical. Não foi possivel explicar as cau. sas destes resultados.

TABELA 4

Resposta à manobra da compressăo do sulco cervical fetal em parturientes, com presença ou ausência de circular cervical do cordão umbilical.

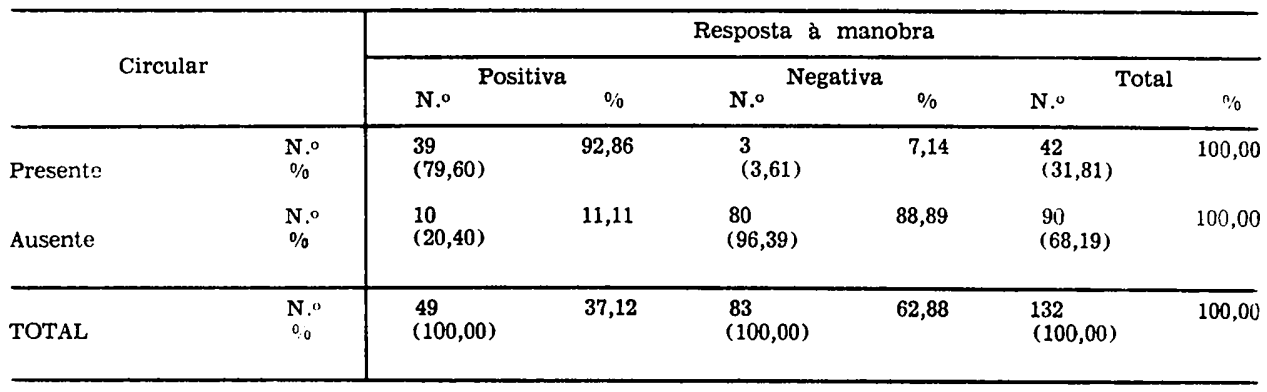

$$
\begin{aligned}
& \qquad \mathrm{x}_{1 \mathrm{gl}}^{2}=81,98 \\
& \text { (nivel de significáncia, } 5 \% \text { ) }
\end{aligned}
$$

Apresentamos na tabcla 5 a influência da paridade na resposta à manobra da compressão do sulco cervical fetal.

Constatamos pelo Teste de Qui-quadrado ${ }^{13}$ que não há diferença significante: entre primíparas e multíparas, isto é, a paridade não influi na resposta positiva ou negativa à manobra aplicada.

TABELA 5

Resposta à manobra da compressão do sulco cervical, positiva ou negativa, em fetos de parturientes

\begin{tabular}{|c|c|c|c|c|c|c|c|}
\hline \multirow{2}{*}{\multicolumn{2}{|c|}{ Paridade }} & \multicolumn{6}{|c|}{ Resposta à manobra } \\
\hline & & \multicolumn{2}{|c|}{ Positiva } & \multicolumn{2}{|c|}{ Negativa } & \multicolumn{2}{|c|}{ Total } \\
\hline & & N.o & $\%$ & N. ${ }^{\circ}$ & $\%$ & N.o & $\%$ \\
\hline Primíparas & $\begin{array}{l}\text { N.o } \\
\%\end{array}$ & $\begin{array}{l}19 \\
(38,78)\end{array}$ & 32,76 & $\begin{array}{l}39 \\
(46,99)\end{array}$ & 67,24 & $\begin{array}{l}58 \\
(43,94)\end{array}$ & 100,00 \\
\hline Multiparas & $\begin{array}{l}\text { N. } \\
\%\end{array}$ & $\begin{array}{l}30 \\
(61,22)\end{array}$ & 40,55 & $\begin{array}{l}44 \\
(53,01)\end{array}$ & 59,45 & $\begin{array}{l}74 \\
(56,06)\end{array}$ & 100,00 \\
\hline TOTAL & $\underset{\%}{N}$ & $\begin{array}{l}49 \\
(100,00)\end{array}$ & 37,12 & $\begin{array}{l}83 \\
(100,00)\end{array}$ & 62,88 & $\begin{array}{l}132 \\
(100,00)\end{array}$ & 100,00 \\
\hline
\end{tabular}
primíparas e multíparas.

Analisando a tabela 6 , verificamos que não há correlação entre a cor do liquido amniótico e a resposta à manobra da compressão do sulco cervical fetal. Constatamos, portanto, que a presença de mecônio não é fator significante, ( $\mathrm{X}^{2}$ $=0,04)$. 
TABELA 6

Cor do líquido amniótico em parturientes que apresentaram resposta positiva ou negativa à manobra da compressáo do sulco cervical do feto.

\begin{tabular}{|c|c|c|c|c|c|c|c|}
\hline \multirow{3}{*}{ Líquido } & \multirow{3}{*}{ Amniótico } & \multicolumn{6}{|c|}{ Resposta à manobra } \\
\hline & & \multicolumn{2}{|c|}{ Positiva } & \multicolumn{2}{|c|}{ Negativa } & \multicolumn{2}{|c|}{ Total } \\
\hline & & N.o & $\%$ & N. ${ }^{\circ}$ & $0 ;$ & N.o & $\%$ \\
\hline \multirow{2}{*}{ Claro } & N. ${ }^{\circ}$ & 31 & 36,47 & 54 & 63,53 & 85 & 100,00 \\
\hline & $\begin{array}{l}\% \\
\text { N.o }\end{array}$ & $\begin{array}{l}(63,27) \\
18\end{array}$ & 38,29 & $\begin{array}{l}(65,0 \\
29\end{array}$ & 6171 & $(64,40)$ & 10000 \\
\hline Meconial & $\%$ & $(36,73)$ & & $(34,9$ & & $(35,60)$ & \\
\hline \multirow{2}{*}{ TOTAL } & N.o & 49 & 37,12 & 83 & 62,88 & 132 & 100,00 \\
\hline & $\%$ & $(100,00)$ & & $(100$ & & $(100,00)$ & \\
\hline
\end{tabular}

A análise da tabela 7 pelo Teste Exato de Fischer ${ }^{12}$ (Fischer $\left.=0,5246\right)$, d(monstra que não há diferença estatisticamente significante da vitalidade do recém-nascido nos cinco primeiros minutos de vida, entre as respostas positivas e negativas à manobra. Em outras palavras, a manobra não prejudica a vitalidade do recém-nascido.

TABELA 7

Correlação da resposta à manobra da compressão do sulco cervical fetal e a avaliação da vitalidade dos 132 recém-nascidos, pelo método de Apgar ,no 5.० minuto de vida.

\begin{tabular}{|c|c|c|c|c|c|c|c|c|}
\hline \multirow{3}{*}{\multicolumn{2}{|c|}{ Método de }} & \multirow{3}{*}{ Apgar } & \multicolumn{6}{|c|}{ Resposta à manobra } \\
\hline & & & \multicolumn{2}{|c|}{ Positiva } & \multicolumn{2}{|c|}{ Negativa } & \multicolumn{2}{|c|}{ Total } \\
\hline & & & N. ${ }^{\circ}$ & $\%$ & N. ${ }^{\circ}$ & $0 ; 0$ & N.o & 00 \\
\hline 0 & $1-1$ & 3 & - & - & - & - & - & - \\
\hline $\begin{array}{l}4 \\
7\end{array}$ & $\square$ & $\begin{array}{r}6 \\
10\end{array}$ & $\begin{array}{r}1 \\
48\end{array}$ & $\begin{array}{r}0,76 \\
36,36\end{array}$ & $\begin{array}{r}3 \\
80\end{array}$ & $\begin{array}{r}2,27 \\
60,61\end{array}$ & $\begin{array}{r}4 \\
128\end{array}$ & $\begin{array}{r}3,03 \\
96,97\end{array}$ \\
\hline \multicolumn{3}{|c|}{ TOTAL } & 49 & 37,12 & 83 & 62,88 & 132 & 100,00 \\
\hline
\end{tabular}

$($ Fischer $=0,5246)$

Analisando os dados apresentados na tabela 8 pelo Teste de Qui-quadrado ${ }^{13}$. observamos que existe correlação entre o comprimento do cordão umbilical e a positividade da resposta à manobra $\left(\mathrm{X}_{1, \mathrm{kl}}^{2}=12,01\right)$. Notamos que nos cordões umbilicais acima de $50 \mathrm{~cm}$ de comprimento há maior possibilidade de respostas positivas à manobra $(97,96 \%)$. Essa correlação é compreensivel, pois, cordões longos predispöem a circulares.

A tabela 9 mostra que há ausência de correlação entre a tensão das circulares cervicais e a positividade da manobra realizada $\left(X_{{ }_{k 1}}^{2}=1,62\right)$, isto é, a porcentagem de manobras positivas ocorrem sem diferença significante entre circulares cervicais frouxas e apertadas.

$\mathrm{Na}$ resposta negativa à manobra aplicada houve $3(10,72 \%)$ circulares cervicais frouxas, não tendo havido caso relacionado com as circulares cervicais apertadas. 
TABELA 8

Correlação entre à resposta da manobra da compressāo do sulco cervical fetal, e o comprimento do cordão umbilical em centímetros.

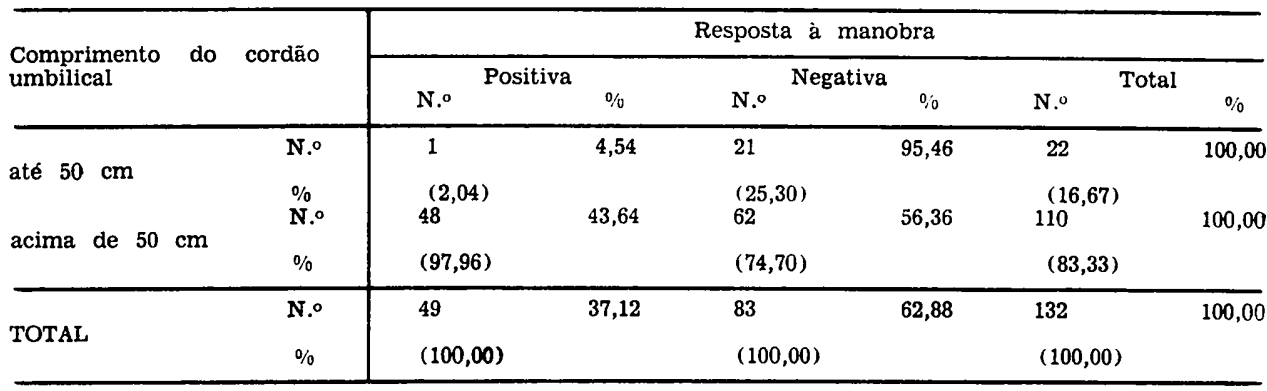

$$
\mathrm{x}_{1 \mathrm{gl}}^{2}=12,01
$$

TABELA 9

Correlação entre a resposta à manobra da compressão do sulco cervical e a tensão da circular do cordão umbilical (frouxa ou apertada), em 42 parturientes.

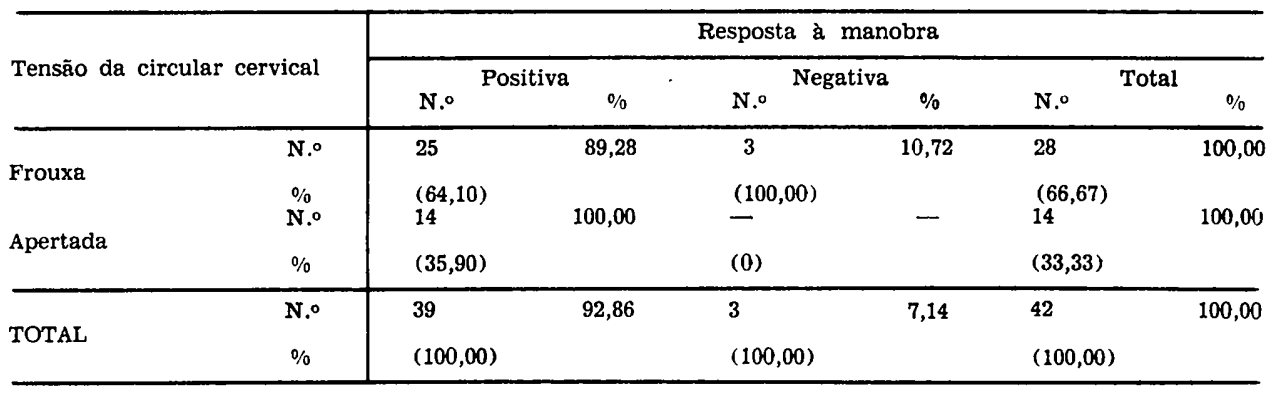

$$
\mathrm{x}_{1 \mathrm{gl}}^{2}=1,62
$$

O estudo estatístico da tabela 10 assinala que não há correlação entre o número de circulares cervicais do cordão umbilical e resposta a manobra da compresão do sulco cervical fetal $\left(\mathrm{X}_{1, \mathrm{q}}^{2}=1,45\right)$. Isto significa que a diferença de porcentagem entre respostas positivas à manobra, com uma circular $(66,67 \%)$ não tem diferença estatisticamente importante da porcentagem de respostas positivas à manobra $(33,33 \%)$ com mais de uma circular cervical.

TABELA 10

Correlação entre a resposta à manobra da compressão do sulco cervical e número de voltas do cordāo

\begin{tabular}{|c|c|c|c|c|c|c|c|}
\hline \multirow{3}{*}{\multicolumn{2}{|c|}{$\begin{array}{l}\text { N.o de circular de cordão } \\
\text { umbilical }\end{array}$}} & \multicolumn{6}{|c|}{ Resposta à manobra } \\
\hline & & \multicolumn{2}{|c|}{ Positiva } & \multicolumn{2}{|c|}{ Negativa } & \multicolumn{2}{|c|}{ Total } \\
\hline & & N. ${ }^{\circ}$ & $\%$ & N.o & $\%$ & N.o & $\%$ \\
\hline \multirow{3}{*}{$\begin{array}{l}\text { uma } \\
\text { mais de uma }\end{array}$} & N.o & 26 & 89,66 & 3 & 10,34 & 29 & 100,00 \\
\hline & \% & $(66,67)$ & 10000 & $(100$, & - & $(69,04)$ & $100 \mathrm{CO}$ \\
\hline & 0.0 & $(33,33)$ & & $(0)$ & & $(30,96)$ & \\
\hline \multirow{2}{*}{ TOTAL } & N. $0^{\circ}$ & 39 & 92,86 & 3 & 7,14 & 42 & 100,00 \\
\hline & 0,1 & $(100,00)$ & & $(100,0$ & & $(100,00)$ & \\
\hline
\end{tabular}
umbilical em torno do pescoço do feto.

$$
x_{1 g l}^{2}=1,45
$$




\section{CONCLUSÕES}

Diante dos fatos apresentados, concluimos o seguinte para csta amostra:

1. Com a manobra de compressão do sulco cervical, aplicada em 132 parturientes, obtivemos $37,72 \%$ de respostas positivas e $62,88 \%$ de respostas negativas. Constatamos a presença de circular cervical em $79,60 \%$ de casos com resposta positiva e em $3,61 \%$ das respostas negativas. Podemos, portanto, considerar que a presente manobra é eficiente para detectar a presença de circular cervical do cordão umbilical.

2. A paridade não influi na resposta à manobra da compressão do sulco cervical, não havendo diferença significativa entre respostas positivis e negativas.

3 . Não há diferença estatisticamente significante (Fischer $=0,5246)$ entre a avaliação da vitalidade do recém-nascido, no $5 .^{\circ}$ minuto de vida, pelo método de Apgar, e a resposta positiva ou negativa à manobra aplicada.

4. Em 49 respostas positivas à manobra da compressão do sulco cervical do recém-nascido, $48(97,96 \%)$ dos cordões umbilicais mediam acima de $50 \mathrm{~cm}$ de comprimento. Há, portanto, correlação entre o comprimento do cordão umbilical e a positividade da resposta à manobra $\left(\mathrm{X}_{1: 1}^{2}=12,01\right)$.

5. Não há correlação entre a tensão das circulares do cordão umbilical (frouxa e apertada) e a resposta positiva à manobra $\left(X_{1 k 1}^{2}=1,62\right)$, ocorrendo o mesmo com o número de circulares cervicais fetal $\left(\mathrm{X}_{1, \mathrm{q} 1}^{2}=1,45\right)$.

6. Em 49 respostas positivas à manobra, obtivemos $10(20,40 \%)$ respostas consideradas falso positivas e em 83 respostas negativas $3(3.61 \%)$, consideradas falso negativas.

MOTTOS. T.: FREDDI, W. E. da S.; RODRIGUES JÚNIOR. N. Manoeuvre of compression of the cervical groove. Rev. Esc. Enf. USP, São Paulo, 15(2):183-192, 1981.

Study about the manoeuvre of compression of the cervical groove, in parturient women, as mean of diagnosis of loops of the umbilical cord, around the fetal neck.

The population was constituted by 132 parturients (58 primiparous and 74 multipa. rous). with a single fetus in cephalic presentation, during the stage of dilatation, with unbroken amniotic membranes and no clinical signs of fetal suffering.

The data obtained give evidence of the efficiency of the manocuvre for dettecting the presence of loops of the umbilical cord around the fetal neck.

\section{REFERENCIAS BIBLIOGRAFICAS}

1. $\triangle$ PGAR, V. A proposal for a new-method of evaluation of the newborn infant. Curr. Res. Anesth., Rocky River, 32 (6): 260-7, Aug./Sept. 1953.

2. ARAMBURU, G. et alii. Obstetrical factors influencing intrapartum compression of the fetal heard and the incidence of dips $I$ in fetal heart rate. Montevideo, Servicio de Fisiologia Obstétrica, Facultad de Medicina, 1969.

3. BARCELLOS, J. M. \& NAHOUM, S. C. Patologia da placenta, das membranas e do cordão umbilical. In: REZENDE, J. Obstetrícia. Rio de Janeiro, Guanabara-Koogan, 1974. cap. 43, p. 854.

4. DE LEE, J. B. \& GREENHILL, J. P. Tratado de obstetrícia. Rio de Janeiro, Guanabara-Koogan, 150 . v. 2, p. 607.8 .

5. GRELLE. F. C. Manual de obstetricia. Rio de Janeiro, Atheneu, 1960. p. 845.6.

6. GREENHILL, J. P. Umbelical cord complications at birth. In: The year book of obstetrics and gynecology. Chicago, Year Book Medical Publishers, 1969. p. 180-1.

7. GULIN, A. L. O sofrimento fetal durante o trabalho de parto. Curitiba, 1969. (Tese de doutoramento - Faculdade de Medicina da Universidade do Paraná). V. 1, p. 553.4; V. 2, p. 346-9.

8. HON, E. H. An atlas of fetal heart rate patterns. New Haven, Harty Press, 1968. p. 164.75.

9. PERES, M. L. Tratado de obstetrícia. Buenos Aires, Lopes S. Etchefoyan, 1951. p. 608-17.

10. PSCHYREMBEL, W. Obstetrícia práctica. 3. ed. Barcelona, Labor, 1978. p. 80.2 e 348.

11. SHAFFER, A. J. \& AVERY, M. E. Doenças do umbigo. In: Lo Doenças do recém-nascido. Rio de Janeiro, Interamericana, 1979. cap. 39, p. 351-9.

12. SIEGEL, S. Estatística não paramétrica. São Paulo, McGraw Hill, 1975. p. 107

13. WALD, A. Sequencial analysis. New York, John Wiley, 1947. 